\title{
Consideraciones sobre la relación entre los Gobiernos, la Iglesia católica y la Universidad de Buenos Aires durante el surgimiento de las universidades privadas confesionales (1955-1958)
}

Jorge Luis Fabián*

Fecha de Recepción: 20 de Enero de 2020

Fecha de Aceptación: 03 de Junio de 2020

DOI: https://doi.org/10.46553/RGES.56.2020.p.68-86

\begin{abstract}
Resumen:
Luego del derrocamiento de Juan Domingo Perón en 1955, comenzó un proceso de "desperonización" que significó, entre otras cosas, la desarticulación del régimen universitario. En tales circunstancias, se promulgó el Decreto de "Organización de las Universidades Nacionales" que pondría fin a la reestructuración y abriría la posibilidad de que la iniciativa privada pudiese crear universidades. En el debate gestado durante el proceso de fundación y reglamentación se observan, principalmente, tres actores: los gobiernos de la "Revolución Libertadora" (1955-1958) y del Dr. Arturo Frondizi (1958-1962), la Iglesia católica y la Universidad.

Debido a que las primeras instituciones en surgir fueron de carácter confesional, comenzarían a entrecruzarse construcciones simbólicas que identificaban la creación de las universidades privadas con un proyecto de la Iglesia. En este contexto, analizaremos algunos de los argumentos propuestos a favor de la creación de estas nuevas universidades y cómo interactuaron los actores mencionados.
\end{abstract}

Palabras clave: Universidad; Catolicismo; Educación

\begin{abstract}
:
After the overthrow of Juan Domingo Perón in 1955, a process of “desperonización” began. That meant, among other things, the dismantling of the university regime. In such circumstances, was promulgated the Decree of "Organización de las Universidades Nacionales" and that would put an end to the restructuring of the peronist model and would conciliate the creation of universities from the private initiative. Three actors would participate in the debate that was generated during the foundation and regulation process: the governments of the "Revolución Libertadora" (1955-1958) and Dr. Arturo Frondizi (1958-1962), the Catholic Church, and the University.

Due to the confessional character of the first universities to emerge, symbolic constructions would begin to intertwine the creation of private institutions with a Church project. In this context, we will analyze some of the arguments proposed in favor of the creation of the new model and how the above mentioned actors interacted during the process.
\end{abstract}

Keywords: University; Catholicism; Education

\footnotetext{
*Universidad del Salvador, jorgelfabian@ @otmail.com
} 


\section{Consideraciones preliminares}

Al producirse el derrocamiento de Juan Domingo Perón en septiembre de 1955 por parte de las Fuerzas Armadas, las instituciones que colaboraron con ellas contarían con su apoyo para llevar a cabo sus proyectos. Tanto la Iglesia católica como las universidades fueron opositores al peronismo durante el proceso previo a la destitución y, por lo tanto, al asumir el nuevo gobierno de facto, intentaron buscar consenso sobre las políticas públicas que las involucraban. En este plano, el gobierno de la autodenominada "Revolución Libertadora" promovió una reestructuración de la universidad que involucró a ambos sectores de diferentes formas. Esta situación habría de provocar una fuerte tensión entre los tres actores involucrados, ya que tanto la Iglesia católica como las universidades comenzaron a cuestionar fuertemente al gobierno, que hasta entonces había intentado satisfacer las demandas de ambos. La necesidad de legitimar el poder que había obtenido por las armas hizo que el gobierno procurara buscar soluciones; las cuales se fueron dilatando hasta que decidió, por lo tanto, dejar en manos del gobierno democrático de Arturo Frondizi la resolución de esta controversia suscitada por la restructuración propuesta. Este proceso concluiría a fines de 1958 con la sanción de una nueva normativa que, sin embargo, no llegaría a satisfacer las expectativas ni de la Universidad ni de la Iglesia pero que, al menos, permitiría sostener buenas relaciones con la última.

Cabe aclarar que, debido a la imposibilidad de abordar en este artículo la situación a escala nacional, analizaremos cómo se produjo la interacción de estos actores en el marco de la Ciudad de Buenos Aires a través del papel de la Universidad de Buenos Aires; mientras que, para las instituciones privadas confesionales se tomarán como ejes a la Universidad Católica Argentina y la Universidad del Salvador.

\section{La reconstrucción de la Universidad de Buenos Aires y el surgimiento de las universidades privadas confesionales}

Con la asunción del Gral. Eduardo Lonardi como primer presidente de la "Revolución Libertadora", se inició un proceso de reconfiguración del régimen universitario que había establecido el gobierno depuesto, y cuyo objetivo era restablecer el principio de autonomía buscando así "reparar el agravio sufrido por la Universidad, sus profesores y alumnos durante la dictadura, y para asentar las bases de reestructuración de su claustro docente y la 
constitución de sus autoridades legítimas". ${ }^{1}$ En este sentido, su primera medida fue intervenir las siete universidades nacionales, cesantear a docentes peronistas y "por primera vez en la historia del siglo se procedió también a la masiva destrucción de libros. Todos ellos favorables al peronismo o simplemente editados por personalidades peronistas". ${ }^{2}$ Con este propósito, se promulgaron los Decretos Leyes 477 y 478 donde se restituía la denominada "Ley Avellaneda" (Ley 1.587/85), y se autorizaba provisoriamente a los interventores a nombrar docentes hasta que se reglamentara la convocatoria a concursos.

Este proceso sería conocido como la "restauración reformista" dado que quienes impulsaron el cambio desde la universidad sostenían los ideales de la Reforma Universitaria de 1918, y en su nombre habían luchado contra el régimen impuesto durante el peronismo. La alianza tácita entre el movimiento estudiantil -representado en el caso de la Universidad de Buenos Aires (UBA) por la Federación Universitaria de Buenos Aires (FUBA)- y el nuevo gobierno, se puso de manifiesto en la designación del Dr. José Luis Romero como Rectorinterventor de la UBA. Este fue electo a partir de una terna propuesta al Poder Ejecutivo por la $\mathrm{FUBA}^{3}$ con la intención, según sostendría el propio Romero, de "que no pareciera una presión aun estando ya resuelto que yo iba a ser designado, es decir cuando ya Lonardi había dado su consentimiento, el ministro Dell’Oro Maini le pidió a la FUBA una terna". ${ }^{4}$ Asimismo, en una declaración del 23 de septiembre de 1955, la Federación Universitaria Argentina (FUA) expresaba "que los estudiantes argentinos han saludado la caída de un régimen opresor y falaz que intentó conculcar todo vestigio de democracia, sumiendo al país en un caos que corrompió la enseñanza primaria y secundaria y destruyó la universidad", ${ }^{5}$ y afirmaba que comenzaba "una nueva etapa de lucha del estudiantado argentino por la universidad autónoma, la enseñanza laica y verdaderamente gratuita y la libertad de cátedra". ${ }^{6}$ Esta particular situación, demostró hasta qué punto el gobierno advertía la existencia de un estudiantado que no se proponía combatirlo y que, al menos públicamente, sostenía ideales similares a los propuestos por la revolución en cuanto a la reestructuración universitaria.

Ahora bien, a pesar del desplazamiento de Lonardi en el mes de noviembre, las nuevas

\footnotetext{
${ }^{1}$ Ley de Organizaciones de Universidades Nacionales, Boletín Oficial de la República Argentina, martes 3 de enero de 1956, año 64, $N^{\circ} 18.059$, p. 1.

${ }^{2}$ Augusto Pérez Lindo, Universidad, política y sociedad (Buenos Aires: EUDEBA, 1985), p. 130.

${ }^{3}$ La terna estaba integrada además por José Babini (nombrado Decano-interventor de la Facultad de Ciencias Exactas y Naturales de la UBA) y Vicente Fatone (nombrado Rector-interventor de la Universidad Nacional del Sur, fundada en enero de 1956).

${ }^{4}$ Félix Luna, Conversaciones con José Luis Romero. Sobre una Argentina con historia, política y democracia (Buenos Aires: Sudamericana, 1986), p. 141.

${ }^{5}$ Alberto Ciria y Horacio Sanguinetti, La Reforma Universitaria (1918-1983) (Buenos Aires: Centro Editor de América Latina), p. 138.

${ }^{6}$ Alberto Ciria y Horacio Sanguinetti, La Reforma Universitaria ..., p. 139.
} 
autoridades presididas por el Gral. Pedro E. Aramburu siguieron los mismos lineamientos ya que el objetivo era continuar con el proceso de "desperonización" y promulgar una nueva ley que reglamentara la actividad de las universidades. Una de las formas en que se manifestó esta continuidad fue la permanencia en el cargo del Ministro de Educación Dr. Atillio Dell`Oro Maini, quien se identificaba con la corriente nacionalista católica a la que adhería el expresidente. Aunque existía una fuerte vinculación entre el ministro y los sectores tradicionales antireformistas que habían participado de la política universitaria durante varios años, incluso en una primera etapa del peronismo, Tulio Halperín Donghi apunta que a partir del reconocimiento de la importancia de los grupos renovadores estudiantiles por parte de Dell'Oro Maini, se empezaba a percibir un cambio en cuanto a las soluciones que se proponían. Asimismo, afirma que aquella política fue la más coherente ya que permitió a la Universidad orillar ciertas crisis particularmente graves, que en el clima pos revolucionario corrían riesgo de extenderse, al tiempo que posibilitó que la tarea de reconstrucción universitaria fuera menos difícil de lo que se hubiera esperado. ${ }^{7}$

En virtud de dicho reordenamiento, y para salvar algunos inconvenientes que ocasionaba el restablecimiento de la "Ley Avellaneda", en los meses de octubre y noviembre, el gobierno resolvió promulgar diferentes normativas donde, entre otras cosas, se fijaban las facultades de los interventores y se los autorizaba, a designar, -conforme a la propuesta de los Delegados Interventores de cada facultad-, a profesores titulares, adjuntos o auxiliares con carácter interino, así como también, a remover o separar profesores cuando lo exigieran los intereses de la reconstrucción universitaria, sin necesidad de previa aprobación por parte del Poder Ejecutivo. ${ }^{8}$ Finalmente, el 23 de diciembre se firmó el Decreto Ley 6403 de Organización de las Universidades Nacionales que puso fin a este proceso de reestructuración. La nueva normativa, les otorgaba a las universidades un régimen jurídico de autarquía para que pudieran dictar sus propios estatutos, y cumplir con la triple finalidad en el orden de la profesión, de la investigación científica y de la universalidad de la cultura. Como se anticipó, podrían elegir y remover a sus profesores sin intervención del Poder Ejecutivo, y además, expedir los certificados de competencia correspondientes a los estudios realizados. ${ }^{9}$

Sin embargo, el mencionado Decreto-Ley presentaba un artículo polémico dado que no se enmarcaba en los objetivos que el gobierno había manifestado. El mismo establecía que

\footnotetext{
${ }^{7}$ Tulio Halperín Donghi, Historia de la Universidad de Buenos Aires (Buenos Aires: EUDEBA, [1962] 2013), p. 156.

8 Decreto-Ley 4361/55: "Facultades de los Interventores en las Universidades Nacionales", http://servicios.infoleg.gob.ar/infolegInternet/anexos/295000-299999/296776/norma.htm

${ }^{9}$ Decreto-Ley 6403/55: "Ley de Organizaciones de Universidades Nacionales". Boletín Oficial de la República Argentina, martes 3 de enero de 1956, año 64, N¹8.059, p. 1.
} 
"la iniciativa privada puede crear universidades libres que estarán capacitadas para expedir diplomas y títulos habilitantes siempre que se sometan a las condiciones expuestas por una reglamentación que se dictará oportunamente". ${ }^{10}$

De manera que esta inclusión del art. 28 que habilitaba el surgimiento de universidades privadas sembró grandes tensiones en distintos sectores de la sociedad argentina, y en particular, generó el rechazo de las agrupaciones reformistas que se oponían, más vigorosamente, a que estas nuevas instituciones otorgaran títulos habilitantes para el ejercicio profesional. Aunque en algunos ámbitos académicos se había planteado la necesidad de la aparición de institutos de formación independientes de la dirección y recursos del Estado, no se esperaba que estos emergieran de una ley, sino que se gestaran como centros de investigación científica y, a partir de su desarrollo, devinieran en universidades. Un ejemplo de esta propuesta era el Instituto de Biología y Medicina Experimental (IBYME) fundado por el Dr. Bernardo Houssay y sus colaboradores al ser expulsados de la UBA durante la "Revolución del '43".

En estas circunstancias, la Iglesia católica se presenta como un actor alineado con el gobierno que intenta aprovechar el contexto de reestructuración del sistema universitario para lograr uno de sus anhelados proyectos: crear su propia universidad. Desde esta perspectiva, José María Ghio sostiene que esto se debió en parte a que, al producirse el alejamiento de Lonardi, la jerarquía eclesiástica, que había imaginado un posible retorno de la obligatoriedad de la enseñanza religiosa en el ámbito de las escuelas públicas, ahora sabía que aquella era una batalla perdida de antemano; por lo que las estrategias viraron hacia la problemática universitaria. ${ }^{11}$ A propósito, recordemos que la única tentativa concreta de fundar una universidad privada en el país, había sido en 1909, cuando el Episcopado Argentino, en su Tercera Reunión Trienal, anunciaba la fundación para el siguiente año de la Universidad Católica de Buenos Aires: "De ninguna manera celebraremos mejor el centenario de nuestra gloriosa independencia que abriendo, el 25 de Mayo de 1910, la Facultad de Derecho y Ciencias Sociales, a la cual irán agregándose a medida que los recursos lo permitan, las varias facultades que debe alcanzar la Universidad Católica". ${ }^{12}$ Sin embargo, esta institución se vería obligada a cerrar sus puertas diez años más tarde, debido a la negativa del Estado para que pudiera expedir los títulos para el ejercicio profesional de sus egresados.

\footnotetext{
${ }^{10}$ Decreto-Ley 6403/55..., p. 2.

${ }^{11}$ José María Ghio, La Iglesia católica en la política argentina. (Buenos Aires: Prometeo, 2007), p. 165.

12 Pastoral Colectiva acerca de la fundación de la Universidad Católica.Tercera Reunión Trienal del Episcopado Argentino. 12 de mayo de 1909. [En línea] http://www.episcopado.org/documentos.php?area=1\&tit_gral=Documentos\%20hist\%C3\%B3rics
} 
Por otra parte, gracias al importante espacio otorgado por el gobierno a los sectores reformistas que integraban la FUBA y que habían apoyado la "Revolución", la Iglesia comenzó a plantear que la UBA venía experimentando un "giro hacia la izquierda". Para dar un ejemplo, la revista Estudios de la Compañía de Jesús reprochaba al gobierno de la universidad "la liviandad con la que se habían echado a los profesores sin tener en cuenta en muchos casos su idoneidad o simplemente por ser católicos, o por no ser lo suficientemente liberal, izquierdista o socialista". ${ }^{13}$

Esta postura crítica, y la firme intención de tener sus propias instituciones educativas en el nivel superior repercutieron, en suma, en la rápida creación de universidades privadas de carácter confesional, las cuales se asentarían sobre estructuras preexistentes. En la Ciudad de Buenos Aires surgieron, entonces, la Universidad Católica Argentina fundada por el Episcopado sobre la base del Instituto Católico de Cultura de Buenos Aires (ICCBA), ${ }^{14}$ y los Institutos Universitarios del Salvador que provenían del Instituto Superior de Filosofía que había fundado la Compañía de Jesús en el Colegio del Salvador. ${ }^{15}$ Además, en algunas provincias proliferaron diferentes institutos que sentarían las bases para las futuras universidades. Algunos de los más significativos fueron: el Instituto Pro Universidad Católica de Córdoba, ${ }^{16}$ fundado "con el más amplio auspicio del Arzobispado de Córdoba, actual Administrador Apostólico Mons. Dr. Fermín Lafitte y la colaboración decidida, a invitación de dicho prelado de la Compañía de Jesús"; ${ }^{17}$ el Instituto Libre Pro Universidad Católica de Santa Fe, ${ }^{18}$ promovido por Mons. Dr. Nicolás Fasolino; el Instituto Pro Universidad Católica de La Plata, ${ }^{19}$ fundado por el Mons. Dr. Antonio Plaza sobre la estructura del Instituto Superior de Cultura Religiosa (derivado de los Cursos de Cultura Católica); y el Instituto

\footnotetext{
13 “Así se traiciona la Revolución”, Estudios, №474 (1956), p. 53.

${ }^{14}$ Luego del cierre de la Universidad Católica de Buenos Aires, la jerarquía eclesiástica junto a un grupo de jóvenes intelectuales católicos fundó los Cursos de Cultura Católica (CCC) que en 1953, bajo la Dirección del Cgo. Luis María Etcheverry Boneo, cambió su nombre por el de ICCBA.

${ }^{15}$ En junio de 1944, la Compañía de Jesús funda el Instituto Superior de Filosofía, el cual pasa a constituirse como Facultad Universitaria recién en 1954. Desde 1956, los distintos Institutos creados pasan a llamarse Facultades, y el 2 de mayo nace la Universidad del Salvador que es denominada - desde el 15 de ese mismo mescomo Institutos Universitarios del Salvador.

${ }^{16}$ Fue fundado el 8 de junio de 1956 por el Arzobispado de Córdoba y otorgado para su dirección a la Compañía de Jesús; lo que ponía en evidencia la unión de dos sectores de la Iglesia. Ese mismo año se abrieron las carreras de Ingeniería, Medicina, Filosofía y Derecho en el ex colegio jesuítico San José. Su primer Rector fue el R.P. Jorge Camargo S.J., y obtendría personería jurídica como universidad en agosto de 1959.

17 “Córdoba docet”, Estudios, No 456 (1956), p. 93.

${ }^{18}$ Fue fundado por el Arzopispo de Santa Fe, Mons. Nicolás Fasolino el 9 de junio de 1957 siendo su primer rector el Cgo. Jorge Funoll. En abril de 1959 comenzaron los cursos regulares de las carreras de Historia y Letras, y su personería jurídica como universidad sería alcanzada en agosto de 1960.

${ }^{19}$ El Instituto Pro-Universidad Católica de la Plata no se consolidó debido a la posibilidad de que la UCA instaurase una Sede en la ciudad de La Plata. Finalmente, el 6 de abril de 1964 se fundó la "Universidad Comunitaria y Católica" (UCOYCA) la cual cambiará su nombre en abril de 1966, por auto arzobispal, por el de Universidad Católica de La Plata (UCALP). El 11 de agosto de 1971 recibirá su autorización definitiva.
} 
Universitario Santo Tomás de Aquino ${ }^{20}$ en Tucumán.

En otro orden de cosas, la creación de estos nuevos espacios hizo que los cuestionamientos no giraran en torno al rechazo a las universidades privadas y la defensa de la institución pública, más bien, el eje del problema se orientaba a una cuestión de índole religiosa y anticlerical. La aparición de un sistema privado de enseñanza superior de tipo confesional, en efecto, chocaba con la tradición educativa argentina basada en el predominio de la instrucción pública y laica, razón por la cual se acusaba al gobierno de fomentar la división de la sociedad sobre parámetros culturales. Esto generó tal tensión que comenzarían a trenzarse construcciones simbólicas al punto de dividir a los protagonistas de esta contienda en "Laicos y Libres". Los primeros, mantendrían una postura crítica hacia la Iglesia acusándola de sectaria y elitista; mientras que los segundos, buscarían defender su propuesta a partir del principio de "libertad de enseñanza" y el cuestionamiento al monopolio de la educación universitaria por parte del Estado, tras el objetivo de desprenderse de la relación "Universidad Privada-Iglesia".

En este camino, podemos observar cómo rápidamente la revista católica Criterio, "que tenía por meta difundir el 'sano' criterio católico en la interpretación de la realidad política nacional e internacional", ${ }^{21}$ comienza a poner atención en la problemática de la libertad de enseñanza y en la importancia de la creación de universidades privadas. El primer número de febrero de 1956 comienza con un editorial de su director Mons. Gustavo Franceschi, titulado "Enseñanza Libre”, y en el número siguiente aparece otra nota del mismo autor con el título de "Universidades Libres". En esta última, se ocupó de refutar las opiniones que José Luis Romero había vertido en una entrevista publicada el 12 de ese mismo mes por el diario $\mathrm{La}$ Nación. Romero, entre otras cosas, había manifestado que la llamada "enseñanza libre" era exactamente igual a lo que en otros tiempos había sido el problema de la enseñanza religiosa. A partir de esto, Mons. Franceschi planteó la necesidad de la creación de universidades libres en provecho de una pluralidad de pensamientos y de la elección de diversos tipos de enseñanza; por lo cual la iglesia se presentaba como uno de los ámbitos que podía abrir sus propias instituciones, aunque no el único. A su vez, sostenía que había una contradicción en el discurso que criticaba la nueva normativa ya que sus detractores recriminaban "al régimen de

\footnotetext{
${ }^{20}$ En 1948 surgen en San Miguel de Tucumán los Cursos de Cultura Católica que en 1950 se convertirían en Cursos de Filosofía Tomista. A partir de estos, se fundará en junio de 1956 el Instituto Universitario Santo Tomás de Aquino (IUSTA). El 16 de mayo de 1959, Mons. Juan Carlos Aramburu, arzobispo de la Arquidiócesis de Tucumán, aprobó los estatutos correspondientes, y se nombrará primer rector a Fr. Alberto Quijano O.P. En agosto de 1965, obtendría el reconocimiento del Poder Ejecutivo Nacional a partir del cual pasaría a denominarse "Universidad del Norte Santo Tomás de Aquino" (UNSTA).

${ }^{21}$ Miranda Lida, Historia del Catolicismo en la Argentina entre el siglo XIX y el XX. (Buenos Aires: Siglo XXI, 2015), p. 120 .
} 
Franco el que mantenga acaparada las Universidades españolas y se califica semejante estado de monstruosa tiranía cuando siguiendo esta posición no vacila en afirmar que el propuesto por los laicistas en este momento argentino le es estrictamente equivalente". ${ }^{22}$

Como ya mencionamos, no solo la apertura de establecimientos universitarios privados era cuestionada, sino especialmente, que estas nuevas instituciones tuvieran la facultad de emitir títulos habilitantes para el ejercicio de las profesiones respectivas. El primer Rector electo de la U.B.A. luego de la intervención propuesta por la "Revolución Libertadora”, Dr. Risieri Frondizi, sostuvo que “(...) el Ministro de Educación doctor Atilio Dell’Oro Maini preparó cuidadosamente el acto. Consultó la casi totalidad del decreto con los rectores interventores, con una doble excepción: el art. 28 y el rector interventor doctor José Luis Romero". ${ }^{23}$

Sin embargo, en este punto vale decir que autores como Emilio Mignone no cuestionan la actitud del ministro, ya que advierten en el citado Decreto-Ley el otorgamiento de reivindicaciones que dos de los sectores que habían apoyado a la revolución en contra del peronismo. Por una parte, el texto establecía "la máxima autonomía para los establecimientos universitarios nacionales, donde eran mayoría las corrientes representativas de la tradición liberal, laica [...] y por otra, se reconoce, con la posibilidad de autorizar universidades privadas, fundamentalmente de orientación confesional, las aspiraciones de la Iglesia católica". 24

Por tanto, fue en este contexto de incertidumbre frente la intencionalidad del problemático artículo, que el gobierno del Gral. Aramburu habría de convocar a fines de febrero de 1956 a la Junta Consultiva Nacional ${ }^{25}$ para que citase al Ministro de Educación con el objetivo de que brindara explicaciones sobre los fundamentos y las intenciones del mentado Decreto-Ley y, puntualmente, de su art. 28. Así, en su exposición el ministro va a sostener que la oportunidad de que la iniciativa privada funde sus propias instituciones "obedece, obviamente, a una concepción acerca de la cultura y acerca de lo que es intrínsecamente la universidad. Pero también, responde a una experiencia histórica y, además, a un anhelo que se

\footnotetext{
${ }^{22}$ Mons. Gustavo Franceschi, “Universidades Libres”, Criterio, año 28, N¹254 (1956), p. 125.

${ }^{23}$ Horacio Sanguinetti, "Historia y régimen de las universidades privadas argentinas", Separata de la Revista de la Universidad Nacional de Córdoba, año VI, marzo-junio (1965), p. 201.

${ }^{24}$ Emilio Mignone, Política y Universidad. El Estado legislador (Buenos Aires: Lugar Editorial,1998), pp. 3637.

${ }^{25}$ Había sido creada en octubre de 1955 con el objetivo de asesorar "al Gobierno Provisional en los problemas relacionados con su gestión y estará compuesta por veinte miembros, representativos de distintas corrientes de opinión", http://servicios.infoleg.gob.ar/infolegInternet/anexos/295000299999/296753/norma.htm
} 
ha abrigado desde hace muchísimos años en el país". ${ }^{26}$ En relación a las opiniones que admitían que la universidad libre tuviera un sentido o una intención confesional, el ministro explicaba que en su espíritu no había entrado jamás esa preocupación y, agregaba que no comprendía en qué medida podía tener ese carácter. ${ }^{27}$ A su vez, el ministro informaba a la Junta acerca de la promulgación del Decreto 3.218, firmado cuatro días antes, donde se había creado una comisión de académicos "con el objeto de asesorar al Gobierno sobre la existencia y funcionamiento de las Universidades libres". La misma, se hallaba integrada por Bernardo Houssay (presidente), Eduardo Braun Menéndez, Alfredo Casares, Venancio Deulofeu, Eduardo Huergo, Teófilo Isnardi, Luis Leloir, Juan T. Lewis, Jaime Perriaux (secretario), Jorge Sábato, José Manuel Saravia, Sebastián Soler y Mauricio Luis Yadarola. ${ }^{28}$ Es importante destacar que, aunque algunos apoyaban la creación de universidades privadas, como era el caso de Braun Menéndez, la postura resultaba moderada ya que si bien alentaba su formación, consideraba que dichas instituciones sólo cobrarían sentido en la medida en que se basaran en la investigación para la creación de conocimiento y no promovieran únicamente la formación profesional. Por cierto, en una entrevista el Dr. Mariano N. Castex -entonces seminarista de la Compañía de Jesús y partícipe junto a su padre, Mariano R. Castex, del proceso de surgimiento de las universidades privadas- afirmaría que "Braun Menéndez jugó políticamente en contra de las universidades privadas, debido a que era de los perfeccionistas y se oponía a estas porque decía que había que tener capital y excelencia, había que partir del núcleo de la plata para bajar como era el modelo de las universidades norteamericanas". ${ }^{29}$

Por otra parte, en aquella reunión con la Junta Consultiva quedaba al descubierto que el surgimiento de universidades libres no era el eje de la controversia. Como manifestó el consejero Américo Ghioldi al propio ministro, la Junta no rechazaba de plano la iniciativa privada; más bien, se oponía a: “(...) la capacidad para expedir diplomas y títulos habilitantes. Aún cuando se ponga la condición de que se sometan a la reglamentación, en definitiva, será ella la que otorgaría el título". ${ }^{30}$

\section{La libertad de enseñanza, los títulos habilitantes y la Universidad de Buenos Aires}

Tal como hemos señalado, el principio de libertad de enseñanza se constituyó en

\footnotetext{
${ }^{26}$ Ministerio de Educación y Justicia, La Revolución Libertadora y la universidad 1955 -1957 (Buenos Aires: Poder Ejecutivo Nacional, 1958), p. 107.

${ }^{27}$ Ministerio de Educación y Justicia, La Revolución Libertadora..., p. 109.

${ }^{28}$ Decreto 3218: "Universidades Libres". Boletín Oficial de la República Argentina, lunes 5 de enero de 1956, año 64, N¹8.193, p. 1.

${ }^{29}$ Entrevista realizada al Dr. Mariano N. Castex, el miércoles 11 de mayo de 2016.

${ }^{30}$ Ministerio de Educación y Justicia, La Revolución Libertadora..., p. 161.
} 
emblema de la campaña a favor de la creación de las universidades privadas por parte de la Iglesia, lo que produjo una ruptura con el tradicional argumento de que las leyes educativas laicistas socavaban las bases de un Estado católico y fomentaban el liberalismo; argumento que oportunamente había sido utilizado para sustentar la necesidad de promover enseñanza religiosa en las escuelas: "Por el contrario, afirmaron que, en base al pluralismo y la diferencia, y aunque los católicos no fueran mayoría, tenían derecho a construir un sistema educativo alternativo". ${ }^{31}$ Este fundamento fue esgrimido desde dos ángulos: el filosófico y el jurídico.

En cuanto al plano filosófico, podemos señalar como antecedentes dos artículos publicados en la revista Ciencia y $F e^{32}$ del año 1945. El primero, escrito por José N. Güenechea S.J. y titulado "Doctrina católica sobre libertad de enseñanza", y el segundo, "Libertad de enseñanza y enseñanza religiosa según el derecho natural", ${ }^{33}$ firmado por Ismael Quiles S.J. Este último, sería publicado un año más tarde, en forma independiente, como "Libertad de enseñanza y enseñanza religiosa", y reeditado en 1955. Allí, Quiles define la libertad de enseñanza como "una libertad de la que gozan, por derecho natural, tanto los individuos como la familia y las asociaciones particulares e implica la facultad de enseñar y poder trasmitir a los demás hombres la ciencia en todos los órdenes"; ${ }^{34}$ además, considera que el Estado tiene una función supletoria ya que debe "fundar instituciones propias docentes cuando no basten las iniciativas privadas para la educación de los ciudadanos". 35 A partir de esta concepción, la Iglesia alegaría que al impedir a la iniciativa privada que abra sus propias instituciones, el Estado violaba este principio, en tanto que "la libertad de aprender y de enseñar, que radica en el derecho que tiene la persona humana a la verdad, no puede ni debe tener otras limitaciones que las que impone la obligación de respetar el orden público básico y la moral pública necesaria para la convivencia humana, que el Estado debe tutelar, cumpliendo con una función que le es propia". ${ }^{36}$

Por otro lado, desde el punto de vista jurídico la base de la argumentación se apoya en el artículo 14 de la Constitución Nacional que pondera el derecho de "enseñar y aprender". Dado que los padres -según la legislación argentina- tienen prioridad y potestad para orientar

\footnotetext{
${ }^{31}$ José Zanca, Los intelectuales y el fin de la cristiandad 1955-1966 (Buenos Aires: Fondo de Cultura Económica, 2006), p. 120.

${ }^{32}$ Publicación trimestral de las Facultades de Filosofía y Teología del Colegio Máximo San José de la Compañía de Jesús en San Miguel, provincia de Buenos Aires.

${ }^{33}$ Ambos artículos publicados en: Ciencia y Fe, año 2, Nº, julio-septiembre, (1945), pp. 7-62.

${ }^{34}$ Ismael Quiles S.J. Libertad de enseñanza y enseñanza religiosa (Buenos Aires: Estudios, 1955), p. 44.

${ }^{35}$ Ismael Quiles S.J. Libertad de enseñanza..., p. 58.

36 "Declaración de la Comisión Permanente del Episcopado Argentino sobre la Liberad de Enseñanza", 1958, http://www.episcopado.org/documentos.php?area=1\&tit_gral=Documentos\%20hist\%C3\%B3ricos
} 
a sus hijos, el Estado asume la función de controlarlos, y en caso de ser necesario, de eliminar la patria potestad. Por ende, su intervención reviste carácter supletorio; o dicho en otros términos, las familias pueden elegir libremente qué tipo de educación pretenden para sus hijos, sin ser coaccionadas por el Estado. También, se ratifica esta postura a partir de la suscripción por parte de la Argentina a la Declaración Universal de los Derechos del Hombre, establecida por la Organización de las Naciones Unidas. ${ }^{37}$ Allí, en el Art. 26 inc. $3^{\circ}$ se declara que "los padres tendrán derecho preferente a escoger el tipo de educación que habrá de darse a sus hijos." De manera que, con estas razones denuncian, pues, que el país no cumple aquello que firmó.

En relación a este último punto, Halperín Donghi concuerda con la postura de Romero ya citada, planteando que la libertad de enseñanza no tenía relación con el debate sobre la creación de instituciones privadas; antes bien, su implementación parece vinculada a la búsqueda de una "solución de compromiso frente a insoportables tensiones preexistentes entre grupos confesionales y laicos", que se volverían a poner de manifiesto en cada grave crisis nacional. ${ }^{38}$ Sin embargo, el entonces Subsecretario de Educación del gobierno de Arturo Frondizi, el profesor Antonio Salonia -y a sesenta años de la sanción de aquel Decreto Leyinsiste en sostener:

“ [...] frente a la innovación de darle protagonismo en el nivel superior a la universidad, no era verdad que este protagonismo tuviera que ver en los propósitos del gobierno solo con el sector católico y la iniciativa que podían concretar los católicos de educación superior. Por eso, dijimos entonces y ratificamos hoy que era falso el dilema de laica o libre, porque el hecho de que pudieran crearse y funcionar universidades privadas no era solamente una prerrogativa, una posibilidad o un camino para el sector católico. También los laicos, de ahí la falsedad del dilema, podían crear universidades privadas". 39

Asimismo, el cuestionamiento a la politización y calidad académica de las universidades nacionales fue un punto neurálgico para justificar la necesidad de la pronta reglamentación de las nuevas instituciones. Recordemos, que durante el peronismo muchos profesores titulares habían sido reemplazados por sus adjuntos o auxiliares, de modo que

37 Resolución 217 A (III), Asamblea General de las Naciones Unidas, 10 de diciembre de 1948, https://www.un.org/es/universal-declaration-human-rights/

${ }^{38}$ Halperín Donghi, La Universidad..., p. 159.

${ }^{39}$ Entrevista realizada al Prof. Antonio Salonia, el lunes 11 de abril de 2016. 
"docentes que habían desempeñado funciones en la Universidad por más de veinte años fueron sustituidos por jóvenes que habían terminado sus carreras hacía menos de un lustro", 40 lo cual fue en detrimento de la calidad académica. Sin embargo, esta situación ya se estaba transformando a partir del llamado a concurso para designar profesores titulares y la elección de las nuevas autoridades.

La crítica por parte de la Iglesia recayó, entonces, en los jóvenes docentes que habían transitado las aulas universitarias durante el peronismo y "en la exclusión de profesores católicos que, a pesar de su reconocida capacidad, han sido alejados de sus cátedras debido a la resistencia organizada contra el pensamiento católico que impedirá que puedan muchas cátedras ser dictadas con criterio católico". ${ }^{41}$ No obstante, este sector también apuntó a los nuevos educadores, a los cuales consideraba "ineptos, y en muchos casos carentes del título universitario necesario, que han sido incorporados en la Universidad, por sus méritos de 'pertenecer a la causa', por su ostensible militancia laicista y por su liberación de prejuicios religiosos". ${ }^{42}$

Si bien la jerarquía eclesiástica era consciente de los problemas que había suscitado la intervención del peronismo en el ámbito universitario, en rigor, lo que cuestionaba era el intento de retorno a una institución politizada, desorganizada por las huelgas y las actividades extraacadémicas; vale decir a una institución cuyos vínculos se remontaban a las transformaciones que había experimentado con la Reforma del '18, y que el "reformismo" buscaba revalidar a través del nuevo Decreto-Ley. Para la Iglesia se estaban dejando de lado los auténticos fines científicos y docentes de la universidad; por lo cual, el nuevo escenario distaba de representar una garantía para la formación de profesionales idóneos y responsables.

Por otro lado, a pesar del fuerte cuestionamiento al régimen universitario establecido por el gobierno peronista, hasta aquel momento la Iglesia no había atacado públicamente dos de sus reformas más emblemáticas: la gratuidad universitaria (1949) y la supresión de los exámenes de ingreso (1953). Sin embargo, luego de la sanción del Decreto-Ley 6403 y con el objetivo de disputar terreno a la universidad, ambas reformas empezarían a ser puestas en discusión.

En relación con la primera, el momento de mayor tensión fue cuando se promulgó la Ley 14.557 en 1958 que dejaría sin efecto el Artículo 28 mediante una nueva disposición que

\footnotetext{
${ }^{40}$ Pablo Buchbinder, Historia de las Universidades Argentinas (Buenos Aires: Sudamericana, 2010), p. 151.

41 "Universidad Católica Argentina", Criterio, año 30, N 1303 (1958), p. 176.

42 "Situación actual de la Universidad Argentina", Sapientia, año XII, N46 (1957), p. 247.
} 
establecía, entre otras cosas, que las universidades privadas recursos estatales. ${ }^{43}$

En cuanto a la segunda, la ley de "Organización de Universidades Nacionales" no modificaba el ingreso irrestricto, y además, en su Artículo $1^{\circ}$ sostenía que las universidades se debían organizar y desenvolver dentro del régimen jurídico de autarquía, teniendo así el pleno gobierno de sus estudios de acuerdo con los estatutos que cada una dispusiera; situación favorable a las universidades nacionales que -con el apoyo de los principales centros de estudiantes- pugnaban por mantener el carácter irrestricto del acceso.

A modo de ejemplo, la revista Criterio también participó de esta polémica para cuestionar al gobierno de la universidad, sosteniendo que el acceso de grandes cantidades de alumnos provocaba fenómenos simétricos de aglomeración y ausentismo que afectaban profundamente la enseñanza. A su vez, exponía el caso del Consejo Directivo de la Facultad de Derecho de la UBA, desde el que se había propiciado la creación de un ciclo básico para el ingreso y se llamaba a concurso para la designación de los profesores respectivos; sin embargo, luego de la reunión del Consejo Superior de la UBA, este "resolvió invitar a la Facultad de Derecho a suspender las diligencias referentes al ciclo básico". ${ }^{44}$ Además, en distintos artículos de esta misma revista se cargan las tintas alrededor de problemáticas como la poca cantidad de alumnos graduados en relación con los que ingresaban, el alto presupuesto que este sistema requería y el progresivo retroceso del nivel académico.

Este último punto, se entronca con la discusión antes señalada acerca del monopolio de las universidades nacionales sobre el otorgamiento de los títulos habilitantes para el ejercicio profesional. Así, quienes denunciaban dicho monopolio encauzaban el cuestionamiento hacia la idoneidad de las universidades estatales para expedir los mismos, y no desde la conveniencia de que los otorgasen también las universidades privadas. De este modo, entonces, planteaban que el dominio de la titulación era evidencia de la capacidad refrendada y controlada por el Estado para el ejercicio de ciertas profesiones cuyas actividades afectaban de modo directo al bienestar público. Por tanto, el Estado se presentaba como el único actor facultado para otorgar dicha habilitación, aunque pudiera también delegarla en otras instituciones como por ejemplo las universidades.

En cuanto a la posición de las Universidades Nacionales, estas manifestaban que desde la primera ley que había reglamentado su funcionamiento (Ley 1597/85) se les transfería esta potestad, al establecerse en su Artículo $1^{\circ}$ inc. 4 "que cada una dará los certificados de exámenes en virtud de los cuales la Universidad expedirá exclusivamente los diplomas de sus

\footnotetext{
${ }^{43}$ Ver: "Ley 14.557”. Boletín Oficial de la República Argentina, 24 de octubre de 1958, Año 66, N¹8.773.

44 “Conflicto en la Facultad de Derecho”. Criterio, Año 30, N 1303 (1958), p. 177.
} 
respectivas profesiones científicas". Aún así, tampoco aceptaban la propuesta de que esta prerrogativa volviera al Estado. No obstante, existían también algunos sectores moderados que podrían aceptar la participación de las instituciones privadas, pero con la condición de que contaran con el aval de una larga y fructífera trayectoria académica. Al respecto, en un discurso brindado en la Facultad de Ingeniería el 9 de septiembre de 1958, el Rector de la UBA, Risieri Frondizi, planteó la necesidad de que no se les concediera el derecho a otorgar títulos habilitantes a las nuevas universidades, debido a que "no han alcanzado el Kindergarten, toda su producción científica cabe en una mano y la totalidad de los alumnos, de todas las universidades privadas reunidas, no alcanza a la quinta parte de los estudiantes que me están escuchando en este momento". ${ }^{45}$ Asimismo, veía absurdo que se pretendieran comparar con instituciones extranjeras que tenían, en muchos casos, siglos de existencia.

\section{La relación entre Frondizi, la Iglesia, y las Universidades privadas}

Como sabemos, a fines de 1957 el gobierno de la "Revolución Libertadora" resolvió convocar a elecciones manteniendo al peronismo proscripto. Por lo tanto, la Unión Cívica Radical (UCR), cuyo electorado había representado aproximadamente al 30\% de la población en todas las elecciones que habían tenido lugar durante el peronismo, el principal beneficiario. Sin embargo, la fragmentación que experimentaba al interior quedaría al descubierto en la convocatoria a elecciones a constituyentes de ese año, puesto que se presentaba escindida en UCR del Pueblo (UCRP), liderada por Dr. Ricardo Balbín, y UCR Intransigente (UCRI) encabezada por el Dr. Arturo Frondizi.

Ahora bien, en la controversia en torno al surgimiento de las universidades privadas, los primeros se manifestaron a favor del monopolio estatal en cuanto a la expedición de los títulos profesionales, e hicieron hincapié en la importancia de la enseñanza laica; mientras que los segundos la implementación de la libertad de enseñanza en todos los niveles a los efectos de obtener un acercamiento con la jerarquía eclesiástica. Por cierto, luego del triunfo de Frondizi en las elecciones del 23 de febrero de 1958, se vería reafirmada tal postura en su discurso de asunción, pues al expresar sus intenciones en el ámbito educativo advirtió: “....el Estado deberá cumplir con los deberes que prescribe la Constitución sobre la libertad de aprender y la libertad de enseñar. Todo argentino debe tener asegurado el acceso a la educación y el derecho de elegir, para sí, o como padre para sus hijos, el tipo de enseñanza

\footnotetext{
${ }^{45}$ Alberto Ciria y Horacio Sanguinetti, La Reforma Universitaria ..., p. 248.
} 
que prefiera". ${ }^{46}$ En consecuencia, apoyándose en las palabras del nuevo presidente, la Iglesia reclamaría a partir de ahora con más ímpetu por la urgencia de la pronta reglamentación del art. 28, debido a que, entre otras razones, sus universidades ya se encontraban en funcionamiento. ${ }^{47}$

Asimismo, existían otros dos elementos importantes para comprender las positivas expectativas de la Iglesia: por un lado, la confirmación como Ministro de Educación del Dr. Luis Mac Kay, quien se había manifestado abiertamente católico y a favor de la libertad de enseñanza; y por otro, la conformación de una comisión -sin darse a conocer públicamentedestinada a elaborar un proyecto de ley que reglamentara el funcionamiento de las universidades privadas, cuyos integrantes eran Ismael Quiles S.J. (Vicerrector de los Institutos Universitarios del Salvador), Aristóbulo Araoz de Lamadrid y Raúl Matera.

Una clara evidencia de la convicción de la Iglesia fue cuando el 7 de marzo - dos meses antes de la asunción de Frondizi-, en el día de la festividad de Santo Tomás de Aquino, patrono de las escuelas católicas, el Episcopado Argentino declaraba oficialmente fundada la Universidad Católica Argentina. ${ }^{48}$ Los estatutos de la nueva universidad serían redactados por una comisión coordinada por el ex Ministro de Educación de la Revolución Libertadora, Dr. Atilio Dell`Oro Maini, por el Dr. Tomás Casares, y por el Mons. Octavio Derisi, quien había sido nombrado Rector de la Universidad. A su vez, en aquella ocasión se proclamó de carácter “necesario seguir reafirmando la conciencia de la libertad de enseñanza en nuestro país, para que $[\ldots]$ pueda cumplir en su futuro próximo, sin trabas legales, la gran misión católica, científica y nacional para la que ha sido creada". ${ }^{49}$

Esta avanzada de la Iglesia, bajo la tutela del gobierno, culminó al hacerse pública, el 11 de junio, la entrega al presidente Frondizi del proyecto de ley de universidades privadas que se le había encomendado a la comisión antes mencionada, concebida para tal fin. ${ }^{50} \mathrm{En}$ este momento, aparecieron fuertemente las Universidades Nacionales a criticar al gobierno y a exigirle la denegación del mentado art. 28. Por este motivo, el Rector de la UBA, Risieri Frondizi daría a conocer un proyecto de ley universitaria que había sido elaborado en

\footnotetext{
46 "Mensaje de Asunción del Presidente Dr. Arturo Frondizi $1^{\circ}$ de mayo de 1958". Dossier Legislativo, Año VI, $\mathrm{N}^{\circ} 152$ (2018), p.79.

${ }^{47}$ A comienzos de 1958 ya funcionaban los Institutos Universitarios del Salvador (Universidad del Salvador) y el Instituto Pro Universidad Católica de Córdoba (Universidad Católica de Córdoba).

${ }^{48}$ El documento fue firmado por el Cardenal Antonio Caggiano, Obispo de Rosario y presidente de la Comisión Permanente del Episcopado Argentino, y refrendado por los treinta y tres Obispos.

49 “Universidad Católica Argentina", Criterio, Año 30, N 1303 (1958), p. 176.

${ }^{50}$ Este "Anteproyecto de la ley para las universidades privadas" fue publicado en su totalidad por la revista Estudios, No498, septiembre (1958), pp.654-59, cuyos autores Ismael Quiles y Raúl Matera contaron con la colaboración de Juan Rodríguez Leonardi, Jorge Clavijo, Aristóbulo Araoz de Lamadrid y María Delia Terrén de Ferro; y con el patrocinio de los Institutos Superiores del Salvador.
} 
Mendoza por todos los rectores de las universidades nacionales, donde establecía que los títulos seguían a cargo, únicamente, de aquellas instituciones. Este proyecto, por consiguiente, entraba en colisión con aquel otro que habían propuesto los convocados por el presidente, en el cual se sostenía que "los titulares de los diplomas académicos, expedidos por las universidades privadas, tendrán el derecho a presentarse al Ministerio de Educación para obtener el reconocimiento de su validez profesional". 51

En este marco, y por iniciativa del diputado de la UCRI, Horacio Domingorena, se discutió en el Congreso Nacional la problemática de las universidades privadas. Oportunamente, el motivo del tratamiento de este tema fue la necesidad de que "el Estado argentino, que ha sido sospechado de totalitario, de monopolista o de enemigo de las libertades, abra sus compuertas, y en circunstancias en las que pudo hacer oídos sordos al reclamo está dispuesto a poner este problema sobre el tapete, a provocar esta ardorosa discusión en su deseo de constituir una universidad privada al servicio del sistema republicano y democrático argentino". 52

Tras extensos debates en ambas Cámaras, y merced a la aplicación de una fuerte “disciplina partidaria” por parte de los representantes de la UCRI, el Senado sancionaría la Ley 14.557/58 de régimen de universidades privadas, que expresaba en su art. $1^{\circ}$ que "la iniciativa privada podrá crear universidades con capacidad para expedir títulos y/o diplomas académicos. La habilitación para el ejercicio profesional será otorgada por el Estado Nacional. Dichas Universidades no podrán recibir recursos estatales". ${ }^{53}$

\section{Breves reflexiones finales}

El proceso de reconstrucción de la universidad que comenzó con la Revolución Libertadora fue el promotor de una nueva estructura universitaria que tuvo como pilares fundamentales las reivindicaciones de las propuestas reformistas y la "desperonización". En el momento en que esto se empezó a llevar a cabo - en parte debido al apoyo a quienes habían derrocado a Perón por parte del movimiento estudiantil- la Iglesia también hizo valer sus buenas relaciones con el gobierno para intentar concretar, tras el fracaso del restablecimiento de la obligatoriedad de la enseñanza religiosa, su viejo anhelo de fundar su propia universidad. En este sentido, se puede entender que el Decreto Ley de Organización de Universidades Nacionales buscara un punto de equilibrio mediante, por un lado, el

\footnotetext{
${ }^{51}$ Ismael Quiles y Raúl Matera, “Anteproyecto de la ley para las universidades privadas” ..., p. 655.

${ }^{52}$ Horacio Domingorena, Diario de Sesiones de la Cámara de Diputados, 24 de septiembre de 1958, Vol. 4, p. 4245 .

53 “Ley 14.557”, Boletín Oficial de la República Argentina, 24 de octubre de 1958, Año 66, ํ⒙773, p. 1.
} 
otorgamiento de la plena autonomía para las universidades nacionales y, por otro, la posibilidad de crear universidades privadas con miras a satisfacer las demandas de la jerarquía eclesiástica.

Quizás, el gobierno no previó que la creación de universidades libres iba a derivar en un conflicto de índole religioso, al reavivar tensiones entre "educación laica o religiosa" que aún estaban latentes. Debido a la necesidad del gobierno de Aramburu de encarar el proceso de "desperonización" y de sortear los inconvenientes que le generaría la aplicación de una nueva política económica de carácter liberal, optó por enfrentar el problema bajo la lógica de “no innovar"; así pues, no derogó el art. 28 pero tampoco lo reglamentó. Su intención era preservar el apoyo que le habían brindado ambos sectores, en virtud de lo cual utilizó a la Comisión Asesora como elemento dilatorio a fin de relegar la cuestión de las universidades privadas al siguiente gobierno constitucional, mientras que las Universidades Nacionales se iban reestructurando bajo la nueva reglamentación.

En un contexto de incertidumbre y con la necesidad de buscar aliados para llegar a la presidencia, Frondizi se acercó a sectores de la izquierda moderada y del peronismo; pero también, comprendió que la reglamentación definitiva de las universidades privadas representaba un momento oportuno para obtener el apoyo de la Iglesia a su candidatura. Por lo además, no contradecía sus convicciones ya que siempre se había manifestado a favor de la implementación de la libertad de enseñanza.

En efecto, en 1958 el gobierno de Frondizi logró sancionar, a pesar de la fuerte oposición de las universidades nacionales y de los sectores laicistas, la mentada ley para regular el funcionamiento de las universidades privadas, sin embargo, la misma no llegó a colmar las expectativas de la Iglesia debido a que no podía la habilitaba para otorgar títulos habilitantes y tampoco, podía recibir financiamiento del Estado. Si bien este conflicto continuó, fue adquiriendo un tenor menos combativo, lo que le permitió al gobierno otorgarle la personaría a las nuevas universidades al año siguiente, y mantener así las buenas relaciones con la Iglesia.

Finalmente, podemos concluir que durante los diferentes gobiernos del período 19551958 la Iglesia actuó, al igual que en los tiempos del peronismo, como factor legitimador ya que necesitaban afianzar su poder frente al pueblo. La característica de este período fue, entonces, la utilización del surgimiento de la universidad privada como contrapartida del apoyo brindado para que se produzca el derrocamiento de Perón, o para la llegada de Frondizi a la presidencia. 


\section{Bibliografía referenciada}

Buchbinder, Pablo. Historia de las Universidades. Buenos Aires: Sudamericana, 2005.

Ciria, Alberto y Sanguinetti, Horacio. La Reforma Universitaria. Buenos Aires: Centro Editor de América Latina, 1983.

Ghio, José María. La Iglesia católica en la política argentina. Buenos Aires: Prometeo, 2007.

Halperín Donghi, Tulio. Historia de la Universidad de Buenos Aires. Buenos Aires: EUDEBA, 2013.

Lida, Miranda. Historia del Catolicismo en la Argentina entre el siglo XIX y el XX. Buenos Aires: Siglo XXI, 2015.

Micheletti, María Gabriela. La universidad en la mira. La Laica o Libre y sus expresiones rosarinas, 1955-1959. Buenos Aires: Imago Mundi, 2013.

Mignone, Emilio. Política y Universidad. El Estado legislador. Buenos Aires: Lugar Editorial, 1998.

Ministerio de Educación y Justicia. La Revolución Libertadora y la universidad 1955 -1957. Buenos Aires: Poder Ejecutivo Nacional, 1958.

Pérez Lindo, Augusto. Universidad, política y sociedad. Buenos Aires: EUDEBA, 1985.

Quiles, Ismael. Libertad de enseñanza y enseñanza religiosa. Buenos Aires: Estudios, 1955.

Sanguinetti, Horacio. "Historia y régimen de las universidades privadas argentinas", Separata de la Revista de la Universidad Nacional de Córdoba, Año VI, N 1-2, marzo-junio (1965): pp. 171-228.

Sanguinetti, Horacio. "Laica o Libre. Los alborotos estudiantiles de 1958”. Todo es Historia, $\mathrm{N}^{\circ} 80$ (1974): pp. 8-23.

Zanca, José A. Los intelectuales católicos y el fin de la cristiandad 1955-1966. Buenos Aires: Fondo de Cultura Económica, 2006.

\section{Fuentes}

Revista Criterio (1955-1958).

Revista Estudios (1955-1958).

Revista Sapientia (1955-1958).

Diario de Sesiones de la Cámara de Diputados. "Organización Universitaria”, 24 de septiembre de 1958, Vol. 4, pp. 4190-4267.

"Mensaje de asunción del Presidente Dr. Arturo Frondizi $1^{\circ}$ de Mayo de 1958". Dossier Legislativo, Año VI, N 152 (2018). [En línea] https://bcn.gob.ar/uploads/Frondizi- 
DOSSIER-legislativoAVIN152-Mensajes-presidenciales.-Mensaje-de-asuncion.-CongresoLegislativo-de-la-Nacion-Argentina.pdf

"Pastoral Colectiva acerca de la fundación de la Universidad Católica".Tercera Reunión Trienal del Episcopado Argentino, 12 de mayo de 1909. [En línea] http://www.episcopado.org/documentos.php?area=1\&tit_gral=Documentos\%20hist\%C3\%B3 $\underline{\text { ricos }}$

Declaración de la Comisión Permanente del Episcopado Argentino sobre la Liberad de Enseñanza, 1958. [En línea] http://www.episcopado.org/documentos.php?area=1\&tit_gral=Documentos\%20hist\%C3\%B3 $\underline{\text { ricos }}$ 